\title{
Linear polarization of hydroxyl masers in circumstellar envelope outer regions
}

\author{
P. Wolak ${ }^{1}$, M. Szymczak ${ }^{1}$ and E. Gérard ${ }^{2}$ \\ ${ }^{1}$ Toruń Centre for Astronomy, Nicolaus Copernicus University, Gagarina 11, \\ 87-100 Toruń, Poland \\ email: wolak@astro.uni.torun.pl \\ ${ }^{2}$ GEPI, UMR 8111, Observatoire de Paris, 5 place J. Janssen, 92195 Meudon Cedex, France
}

\begin{abstract}
A recent polarimetric survey of $\mathrm{OH}$ masers in a large sample of AGB and post-AGB stars revealed widespread occurrence of polarized features. We made a statistical analysis of the polarization properties of this large data set. We discuss the alignment of polarization position angles between the extreme blue- and red-shifted parts of the $1612 \mathrm{MHz}$ spectrum. The average polarization angle of $\mathrm{OH}$ masers from the opposite sides of the envelope agrees within $20^{\circ}$ for $80 \%$ of the sources in the sample. For two objects monitored over $\sim 6$ years the polarization position angle at $1612 \mathrm{MHz}$ is constant within measurement uncertainties: this suggests a stable and a very regular structure of the circumstellar magnetic fields. Alternatively, this could indicate a galactic origin of the field which may be amplified by the stellar wind in the outermost parts of the envelopes.
\end{abstract}

Keywords. masers - polarization - circumstellar matter - magnetic field - stars: AGB and post-AGB

\section{Introduction}

Late-type stars showing high mass loss are known to harbor magnetic fields in their envelopes. The magnetic field strength and its structure have been determined by $\mathrm{SiO}$, $\mathrm{H}_{2} \mathrm{O}$ and $\mathrm{OH}$ maser observations (eg. Kemball \& Diamond 1997, Szymczak et al. 1998, Vlemmings et al. 2005). As these species occur in different parts of the circumstellar envelope it is possible to recover a picture of the magnetic field throughout the entire envelope (Szymczak et al. 2001, Vlemmings et al. 2005, Vlemmings et al. 2011). In the inner regions of the envelope the field strengths are strong enough to dynamically influence the shaping of the stellar wind but it is still debated whether the magnetic field is important in forming asymmetric structures observed in planetary nebulae (Bains et al. 2003, Soker 2006).

Early studies of the $\mathrm{OH} 1612 \mathrm{MHz}$ maser lines in AGB stars claimed a lack of polarized features. Improvements of spectral resolution up to $0.1 \mathrm{~km} \mathrm{~s}^{-1}$ enabled detection circularly polarized features in several objects (Zell \& Fix 1991), whereas linear polarization was usually weak, if any (Olnon et al. 1980). Our recently published polarimetric survey of AGB and post-AGB stars revealed widespread occurrence of polarized $\mathrm{OH}$ maser emission (Wolak et al. 2012). More than $75 \%$ of the objects in the complete sample have polarized features. One of our findings is a very small difference in the position angle of linearly polarized emission from the extreme blue- and red-shifted parts of the $1612 \mathrm{MHz}$ spectra. The standard model of circumstellar envelopes (Reid et al. 1977) predicts that the $1612 \mathrm{MHz}$ outermost peaks come from two very compact regions on the opposite sides of the envelope. Indeed, early interferometric observations fully confirmed this model. For instance the $1612 \mathrm{MHz}$ emission of $\sim 0.5 \mathrm{~km} \mathrm{~s}^{-1}$ width from the near and 
Table 1. Position angles of the linearly polarized emission for two stars. The $\Delta \chi_{1612 \mathrm{~B}-\mathrm{R}}$ is the difference between the averaged polarization position angles for the extreme blue- and red-shifted parts of the $1612 \mathrm{MHz}$ spectrum. The $\Delta \chi_{1667-1612 \mathrm{R}}$ is the difference between the averaged polarization position angles of the red-shifted parts of the 1667 and $1612 \mathrm{MHz}$ spectra. SD is the standard deviation and SDM is the standard deviation of the mean.

\begin{tabular}{llccccc}
\hline Name & \multicolumn{1}{c}{$\Delta$} & Number of epochs & Mean & SD & $\begin{array}{c}\text { SDM } \\
\left({ }^{\circ}\right)\end{array}$ & Median \\
& & & & & \\
\hline OH127.8+0.0 & $\Delta \chi_{1612 \mathrm{~B}-\mathrm{R}}$ & 181 & 1.8 & 8.1 & 0.6 & 3.1 \\
& $\Delta \chi_{1667-1612 \mathrm{R}}$ & & 27.6 & 15.4 & 1.1 & 27.9 \\
OH17.7-2.0 & $\Delta \chi_{1612 \mathrm{~B}-\mathrm{R}}$ & 172 & 2.1 & 3.9 & 0.3 & 1.7 \\
& $\Delta \chi_{1667-1612 \mathrm{R}}$ & & 65.7 & 18.7 & 1.4 & 62.5 \\
\hline
\end{tabular}

far caps of the shell of the archetypal $\mathrm{OH} / \mathrm{IR}$ source $\mathrm{OH} 127.8+0.0$ remained unresolved with a 0.03 " beam (Norris et al. 1984). This implies that the sizes of the extreme sides of the envelope are less than $0.8 \%$ of its diameter. Thus, high spectral resolution spectra of about $0.1 \mathrm{~km} \mathrm{~s}^{-1}$ obtained even with a $3.5^{\prime} \times 19^{\prime}$ beam reliably probe a local magnetic field because the depolarization effect is negligible. We discuss the properties of the linearly polarized emission by adding new data obtained in a monitoring project.

\section{Observations}

The observations were carried out with the Nançay Radio Telescope (NRT) from May 2003 to December 2008. The technical details of the NRT were described by van Driel et al. (1996). All four Stokes parameters were measured at 1612 and $1667 \mathrm{MHz}$ simultaneously. The methods of observation, instrumental polarization calibrations and error budget are discussed elsewhere (Szymczak \& Gérard 2004). The spectral resolution was $\sim 0.14 \mathrm{~km} \mathrm{~s}^{-1}$ or $\sim 0.07 \mathrm{~km} \mathrm{~s}^{-1}$. The system directly provided three of the four Stokes parameters, namely $I, Q$ and $V$, while the fourth parameter $U$ was extracted by a horn rotation of $45^{\circ}$. The linearly polarized flux density $p=\left(Q^{2}+U^{2}\right)^{0.5}$, fractional linear polarization, $m_{\mathrm{L}}=p / I$, and polarization position angle, $\chi=0.5 \tan ^{-1}(U / Q)$, were derived from the Stokes parameters. The properties of the star sample are given in Wolak et al. (2012). In the following we limit the discussion to the sources that have both extreme peaks at $1612 \mathrm{MHz}$ brighter than $10 \sigma$, i.e. about $0.35 \mathrm{Jy}$.

\section{Results}

Linearly polarized features are detected in 59 and 22 sources at $1612 \mathrm{MHz}$ and $1667 \mathrm{MHz}$, respectively. There are $291612 \mathrm{MHz}$ sources with linearly polarized emission in both parts of the double peaked profile. Some examples of $p$ spectra with the superimposed position angle of linear polarization are shown in Fig. 1.

At $1612 \mathrm{MHz}$ most of the sources show only weak $\left(<15^{\circ}\right)$ variations of the polarization angle from channel to channel. The analysis presented in Wolak et al. (2012) is extended here by considering data from multi-epoch observations of 14 sources. The difference between the average polarization position angles for the four most blue- and red-shifted channels in the $1612 \mathrm{MHz} p$ profile, $\Delta \chi_{1612 \mathrm{~B}-\mathrm{R}}$, is calculated. Its value is lower than $20^{\circ}$ for more than $80 \%(24 / 29)$ of the sources and is higher than $45^{\circ}$ for two sources only. For the whole subsample the absolute average and median values of $\Delta \chi_{1612 \mathrm{~B}-\mathrm{R}}$ are $16.2 \pm 2.9$ and $13.9^{\circ}$, respectively. For a few sources observed at several epochs spanning $6-8$ years, no systematic variations of $\Delta \chi_{1612 \mathrm{~B}-\mathrm{R}}$ higher than $10^{\circ}$ are detected.

Nine sources in the sample have linearly polarized emission at the two frequencies from the same usually red-shifted side of the shell. The scatter of $\chi$ angles of the $\mathrm{OH}$ mainline channels is on average 2.7 times greater than that of $1612 \mathrm{MHz}$ lines. For seven 


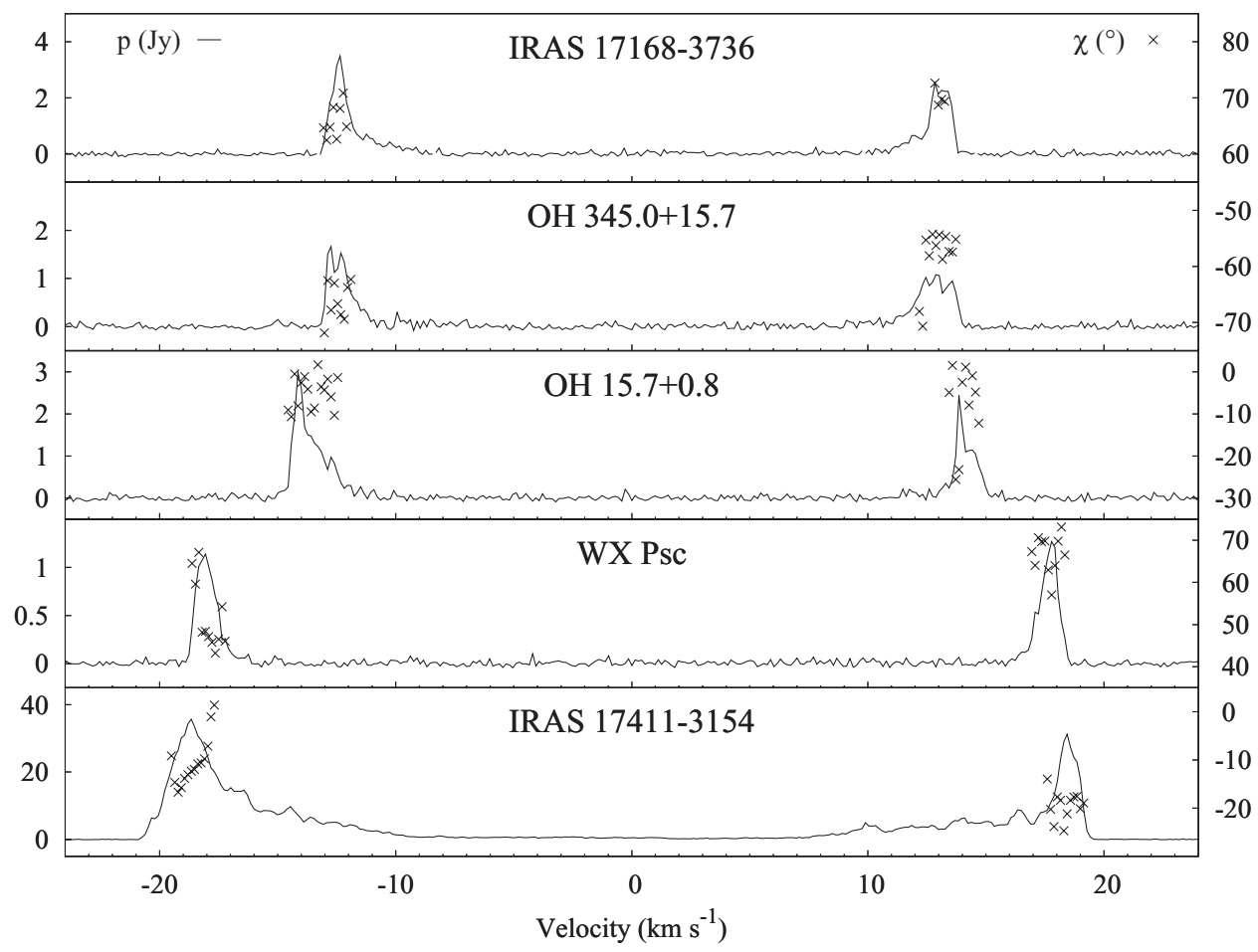

Figure 1. $\mathrm{OH} 1612 \mathrm{MHz}$ spectra of the linearly polarized emission, $p$ of the selected sources with a double peaked profiles. The polarization position angle (points) are superimposed (right ordinate) for the extreme outside channels. The velocity scale is shown with regard to the systemic velocity.

sources, the velocities of the extreme emission at both frequencies overlap within less than $0.1 \mathrm{~km} \mathrm{~s}^{-1}$. In the majority of sources the difference in the average polarization angle for four neighbouring channels of the 1612 and $1667 \mathrm{MHz}$ lines ranges from 36 to $80^{\circ}$. The mean difference for nine sources is $44 \pm 8^{\circ}$ and the median is $47^{\circ}$.

The Stokes $I$ profiles at 1612 and $1667 \mathrm{MHz}$ and the polarization position angle are shown superimposed for two sources only (Fig. 2) for the sake of conciseness. The times series of polarization position angles for the extreme blue- and red-shifted features, their differences at $1612 \mathrm{MHz}, \Delta \chi_{1612 \mathrm{~B}-\mathrm{R}}$, and the differences between average polarization angles for the four red-shifted channels in the 1667 and $1612 \mathrm{MHz} p$ profiles, $\Delta \chi_{1667-1612 \mathrm{R}}$ are also shown. We note that $\Delta \chi_{1667-1612 \mathrm{R}}$ is $2-4$ times more scattered than the $\Delta \chi_{1612 \mathrm{~B}-\mathrm{R}}$ (Tab. 1). For the sources OH127.8+0.0 and OH17.7-2.0 the averages $\Delta \chi_{1612 \mathrm{~B}-\mathrm{R}}$ over $\sim 6$ years are less than 1.8 and $2.1^{\circ}$, respectively (Tab.1). We note a gradual increase of this difference in the latter object which is known to experience secular changes. The scatter of $\chi$ angles within the $\mathrm{OH}$ mainline channels is on average 2.7 times greater than that within $1612 \mathrm{MHz}$ lines. This may suggest that either the $1667 \mathrm{MHz}$ masers come from more turbulent regions and/or the magnetic fields are less ordered.

\section{Discussion}

Small differences in the polarization angles at the near and far edges of the $\mathrm{OH}$ shells in our subsample suggest a regular magnetic field geometry in the $1612 \mathrm{MHz}$ maser regions. 

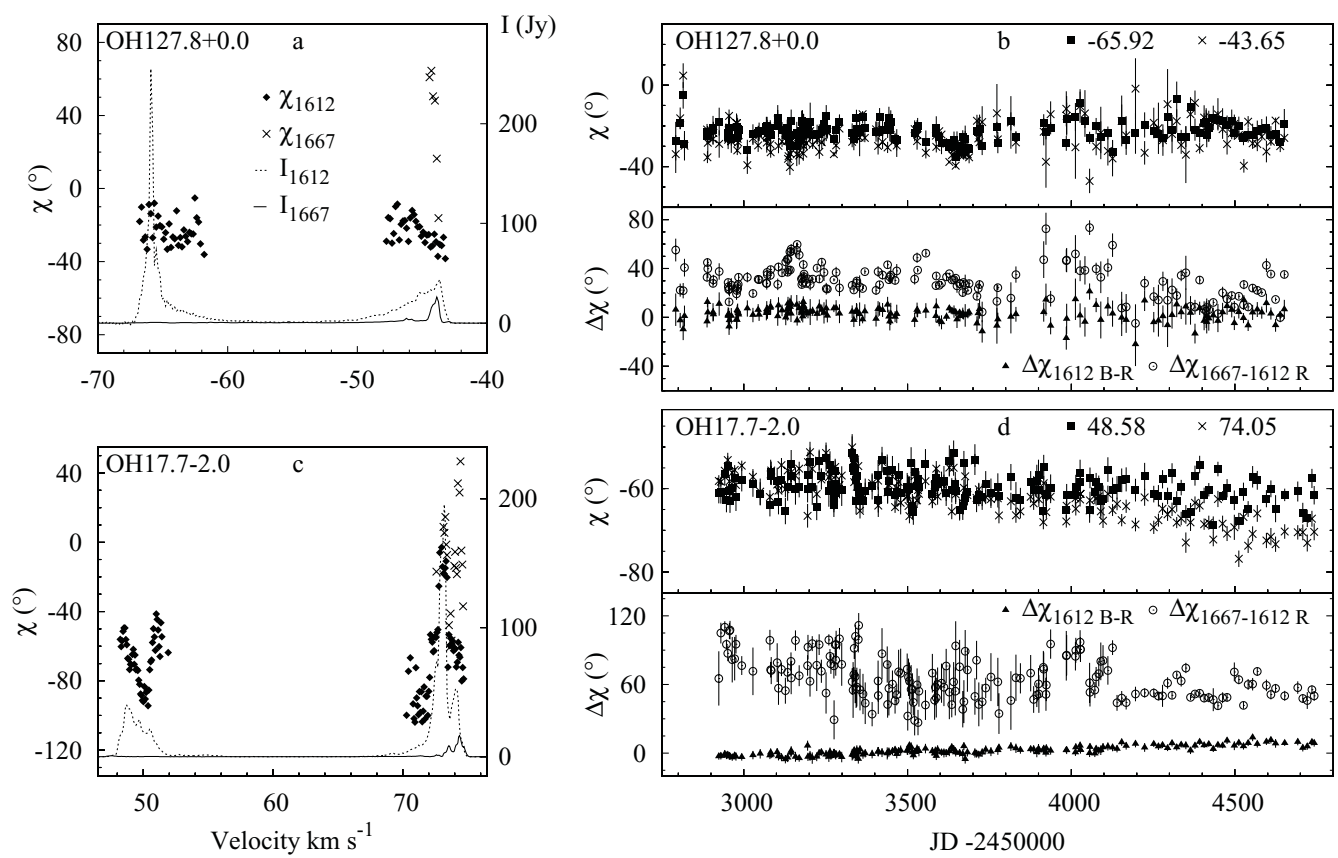

Figure 2. Position angles of the linearly polarized emission in two OH/IR stars. (a) The Stokes $I$ spectra at $1612 \mathrm{MHz}$ (dashed line) and $1667 \mathrm{MHz}$ (solid line) of OH127.8+0.0 with superimposed polarization position angles, $\chi$ marked as diamonds and crosses for 1612 and $1667 \mathrm{MHz}$, respectively. (b) (upper panel) polarization position angle time series for the brightest extreme blue- and red-shifted four-channel features at $1612 \mathrm{MHz}$ and (lower panel) the difference in polarization position angles of these features, $\Delta \chi_{1612 \mathrm{~B}-\mathrm{R}}$ (filled triangles) and the difference in polarization position angles between 1612 and $1667 \mathrm{MHz}$ red-shifted features, $\Delta \chi_{1612-1667 \mathrm{R}}$ (open circles). (c) and (d) are the same as (a) and (b), respectively but for OH17.7-2.0.

However, it is hard to explain such a homogeneous magnetic field over $\mathrm{OH}$ shells of large diameters (e.g. Habing 1996). A plausible explanation for the low values of $\Delta \chi_{1612 \mathrm{~B}-\mathrm{R}}$ is that the origin of the field is intrinsic and the same field orientation is carried away (frozen in) by the stellar wind flowing towards the front and back of the envelope. Alternatively, the magnetic field could be of Galactic origin and be amplified and/or distorted by the stellar wind. The interstellar fields can pervade the outer $\left(10^{16-17} \mathrm{~cm}\right)$ regions of circumstellar envelopes and this hypothesis needs further investigation. High values of $\Delta \chi_{1612 \mathrm{~B}-\mathrm{R}}$ in a minority of sources suggest a deviation from globally ordered magnetic field caused by local outflows or outbursts. The latter possibility is likely for OH17.7-2.0 where $\Delta \chi_{1612 \mathrm{~B}-\mathrm{R}}$ is only $2.1^{\circ}$ but the polarization angle of the eruptive feature near $73 \mathrm{~km} \mathrm{~s}^{-1}$ (Szymczak \& Gérard 2005) is about $-15^{\circ}$ and differs by about $64^{\circ}$ from the mean value for the extreme blue- and red-shifted velocities. The polarization position angles at the extreme parts of the $1667 \mathrm{MHz}$ spectrum are different and their scattering from channel to channel is much higher that those at $1612 \mathrm{MHz}$. This suggests that the $1667 \mathrm{MHz}$ emission probes regions with less regular magnetic fields than those probed by the $1612 \mathrm{MHz}$ line.

\section{References}

Bains, I., Gledhill, T. M., Yates, J. A., \& Richards, A. M. S. 2003, MNRAS, 338, 287

Bowers, P. F. \& Johnston, K. J. 1990, ApJ, 354, 676 
Habing, H. J. 1996, APPR, 7, 97

Kemball, A. J. \& Diamond, P. J. 1997, ApJ, 418, L111

Norris, R. P., Booth, R. S., Diamond, P. J., et al. 1984, MNRAS, 208, 435

Olnon, F. M., Winnberg, A., Matthews, H. E., \& Schultz, G. V. 1980, AAPS, 42, 1190

Reid, M. J., Muhleman, D. O., Moran, J. M., et al. 1977, ApJ, 214, 60

Soker, N. 2006, PASP 118, 260S

Szymczak, M., Cohen, R. J., \& Richards, A. M. S. 1998, MNRAS, 297, 1151

Szymczak, M., Cohen, R. J., \& Richards, A. M. S. 2001, A\& A, 371, 1012

Szymczak, M. \& Gérard, E. 2004, A\&A, 423, 209

Szymczak, M. \& Gérard, E. 2005, A\&A, 433, L29

van Driel W., Pezzani J., Gérard E. 1996, in High Sensitivity Radio Astronomy, ed. N. Jackson, \& R. J. Davis (Cambridge Univ. Press), 229

Vlemmings, W. H. T., van Langevelde, H. J., \& Diamond, P. J. 2005, A\&SA, 434, 1029

Vlemmings, W. H. T., Humphreys, E. M. L., \& Franco-Hernández, R. 2011, ApJ, 728, 149

Wolak, P., Szymczak, M., \& Gérard, E. 2012, A\&A, 537, A5

Zell, P. J. \& Fix, J. D. 1991, ApJ, 369, 506 